\title{
Two different origins of the $Q$-slope problem in superconducting niobium film cavities for a heavy ion accelerator at CERN
}

\author{
A. Miyazaki ${ }^{1,2}$ and W. Venturini Delsolaro ${ }^{1}$ \\ ${ }^{1}$ CERN, 1211, Geneva 23, Switzerland \\ ${ }^{2}$ School of Physics and Astronomy, University of Manchester, Manchester, M13 OPL, United Kingdom
}

(Received 11 December 2018; published 3 July 2019)

\begin{abstract}
Superconducting niobium film cavities deposited on copper substrates $(\mathrm{Nb} / \mathrm{Cu})$ have suffered from strong field-dependent surface resistance, often referred to as the $Q$-slope problem, since their invention. We argue that the $Q$-slope may not be an intrinsic problem, but rather originates from a combination of factors which can be revealed in appropriate environmental conditions. In this study, extrinsic effects were carefully minimized in a series of experiments on a seamless cavity. The origin of the $Q$-slope in low frequency cavities is traced back to two contributions with different temperature and magnetic field dependences. The first component of $Q$-slope, affecting the residual resistance, is caused by trapped magnetic flux which is normally suppressed by a magnetic shield for bulk niobium cavities. The second, temperature dependent component of $Q$-slope, is similar to the medium-field $Q$-slope which is well known in bulk niobium cavities. These results are compared with theoretical models and possible future studies are proposed.
\end{abstract}

DOI: 10.1103/PhysRevAccelBeams.22.073101

\section{INTRODUCTION}

Superconducting radio frequency (SRF) cavities are becoming more and more attractive as a core component of modern accelerators. So far, the technology of choice for most realizations was based on high purity niobium sheets as the raw material for manufacturing the resonators. Niobium film cavities deposited on copper substrates $(\mathrm{Nb} / \mathrm{Cu})$ were introduced as a promising alternative to bulk niobium cavities [1]. The $\mathrm{Nb} / \mathrm{Cu}$ technology not only reduces production costs, but also increases thermal stability thanks to the high thermal conductivity of the film substrate. $\mathrm{Nb} / \mathrm{Cu}$ cavities were adopted for the Large Electron Positron collider (LEP-II), Acceleratore Lineare Per Ioni (ALPI), Large Hadron Collider (LHC), and High Intensity and Energy Isotope Separator On Line DEvice (HIE-ISOLDE) accelerators [2]. All these projects had relatively low field requirements. On the other hand, the gradual increase of surface resistance at high rf field, the so-called $Q$ problem, prevents the adoption of this technology for very high-gradient accelerators, such as the International Linear Collider (ILC). In this work, we experimentally studied $Q \mathrm{~s}$ in a $\mathrm{Nb} / \mathrm{Cu}$ cavity by changing the environmental conditions.

Published by the American Physical Society under the terms of the Creative Commons Attribution 4.0 International license. Further distribution of this work must maintain attribution to the author(s) and the published article's title, journal citation, and DOI.
The conventional description of the surface resistance of superconductors in the Meissner state is based on linear response theory. At sufficiently low fields, the superconductor response can be perturbatively expanded [3], and its first order term gives the so-called Bardeen-CooperSchrieffer (BCS) surface resistance $[4,5]$. When the applied rf frequency is much lower than the superconducting gap energy and the operational temperature is below half of the superconducting transition temperature, the BCS resistance can be approximated as [6]

$$
R_{\mathrm{BCS}}(T)=\frac{A_{0}}{T} \exp \left(-\frac{\Delta_{0}}{k_{\mathrm{B}} T}\right)
$$

where $A_{0}$ depends on material parameters and rf frequency, and $\Delta_{0}$ is the superconducting gap determined by the selfconsistent gap equation of an equilibrium superconductor [3]. A recent study by the authors on the use of this term in cavity data analysis is in Ref. [7].

It must be underlined that the BCS resistance, obtained by linear response theory, is independent of the applied rf field, and, more importantly, that the theory itself is only valid at very low fields, thus the available microscopic formulae do not tell us anything about the behavior of the surface resistance at the rf fields of interest. The $Q$ observed in $\mathrm{Nb} / \mathrm{Cu}$ cavities might be a non-linear effect beyond this microscopic description. If the origin of the $Q$ is intrinsic and has to be explained by a microscopic theory, its study can be a means to examine the nonequilibrium statistical mechanics of quantum field theory, since superconductivity 
is nothing but the macroscopic appearance of quantum effects in a many body system. However, since real cavities for accelerators have huge surfaces, macroscopic inhomogeneities or thermal instabilities could also be invoked to explain the observed nonlinear behavior.

This report is organized as follows. First, the experimental setup and the main features of our cavity design are introduced. Second, we report the raw results of all the measurements carried out. Third, the empirical features of the $Q \mathrm{~s}$ are exposed by a suitable data analysis. Finally, results are discussed in terms of the historical context together with physics implications: some theoretical models proposed previously are excluded or partially supported by our data, suggesting possible future prospects.

\section{EXPERIMENTAL}

Two types of quarter-wave resonator (QWR) [8] were developed for the HIE-ISOLDE project. The original HIEISOLDE cavities for the series production contained a circular electron beam weld, at the top of the copper substrate, connecting inner and outer conductors as shown in Fig. 1(a). This feature induced a delicate problem in cool down dynamics and made these cavities unsuitable for a systematic study of the superconducting properties of the niobium film. The performance of the welded cavities is summarized in Appendix A.

In order to improve the surface quality of the copper substrate and enhance the thermal conductance to the helium bath, we developed a new cavity machined out of a solid copper billet, in which welding processes were avoided as shown in Fig. 1(b). This cavity has axial symmetry, apart from the beam ports, and a virtually defect-free surface in the high-field region. The mechanical design of this seamless cavity is summarized in Ref. [9] and Ref. [10]. In this paper,
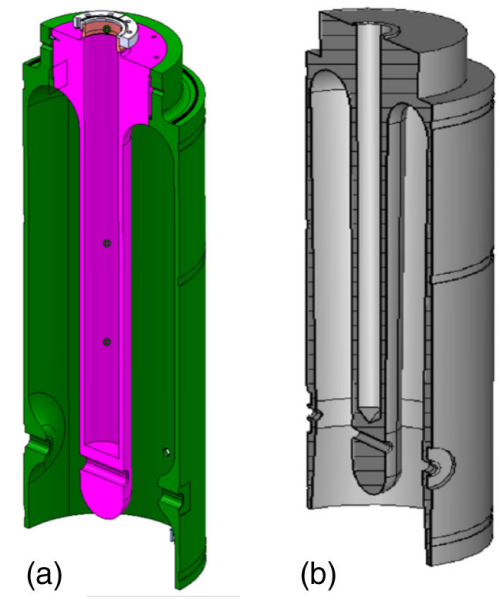

FIG. 1. Cross-section view of (a) the original substrate made of inner (magenta) and outer (green) pieces connected by electronbeam welding, and (b) the seamless substrate machined out of a copper billet. the surface resistance of this niobium-coated seamless QWR was studied.

The resonance frequency of the cavity is $101.28 \mathrm{MHz}$ and the specified power consumption for HIE-ISOLDE is $10 \mathrm{~W}$, corresponding to the surface resistance of $65 \mathrm{n} \Omega$ at $6 \mathrm{MV} / \mathrm{m}$ and $4.5 \mathrm{~K}$.

The accelerating gradient $E_{\text {acc }}$ depends on the definition of effective length of the cavity. In the HIE-ISOLDE project, we conventionally define

$$
E_{\mathrm{acc}}=\frac{\int_{0}^{L}\left|E_{z}(z)\right| d z}{L}
$$

where $E_{\mathrm{z}}$ is the electric field component along the beam axis, and $L=0.3 \mathrm{~m}$ is the inner diameter of the cavity. The transit time factor (TTF) effect was not included in this definition for simplicity. The ratio between $E_{\text {acc }}$ and the peak magnetic field $B_{\text {peak }}$ on the cavity surface is $9.3 \mathrm{mT} /(\mathrm{MV} / \mathrm{m})$.

The intrinsic quality factor $Q_{0}$ of the cavity was evaluated by standard methods [7] with a phase lock loop circuit. The measurement was carried out for different temperatures and rf fields. First, the accelerating field was scanned from $50 \mathrm{kV} / \mathrm{m}$ to $6 \mathrm{MV} / \mathrm{m}$ at $4.5 \mathrm{~K}$. Then, $Q_{0}$ was measured at a fixed field level while the cavity was cooled down to $2.4 \mathrm{~K}$. Although the liquid helium temperature is below $2.0 \mathrm{~K}$, in the bottom part of the cavity, the temperature is at $2.4 \mathrm{~K}$ due to the static thermal resistance of the conduction-cooled copper substrate. At this point, the field was scanned at $2.4 \mathrm{~K}$. Finally, the cavity was warmed up to $4.5 \mathrm{~K}$, and $Q_{0}$ was again measured at another fixed field. This cycle was repeated four times, at different $\mathrm{rf}$ field levels. In a second series of measurements, the same procedure was carried out after a thermal cycle above the critical temperature $T_{\mathrm{c}}=9.5 \mathrm{~K}$, with different ambient magnetic field levels when the cavity crossed $T_{\mathrm{c}}$ during the subsequent cool down. The ambient field was controlled by external coils wound around the cryostat, and it was measured by flux-gate sensors placed around the cavity.

The ambient field of the vertical cryostat was controlled to be $5 \mu \mathrm{T}, 50 \mu \mathrm{T}$, and $100 \mu \mathrm{T}$ for the systematic study of surface resistance. Since the HIE-ISOLDE cryomodule is not equipped with a magnetic shield, the measurement point with the earth field level of $50 \mu \mathrm{T}$ was necessary to estimate its influence on the accelerator performance. Three flux-gate sensors measured the magnetic field in the cryostat, and the spatial distribution of the field is neglected as a second order effect.

\section{RESULTS}

Figure 2 shows the measured $Q_{0}$ as a function of $E_{\text {acc }}$ for a reduced external magnetic field $(5 \mu \mathrm{T})$ upon cool down through $T_{\mathrm{c}}$, and Fig. 3 shows the same measurements after cool down with an enhanced external field $(100 \mu \mathrm{T})$. These 


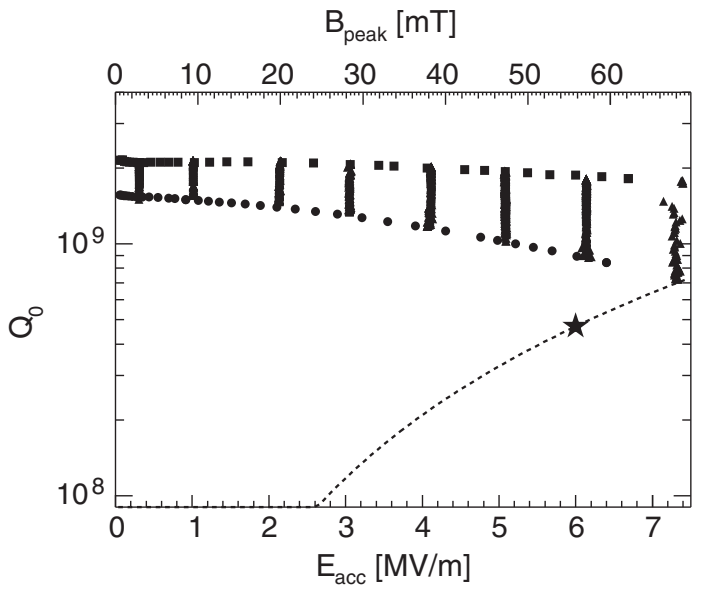

FIG. 2. Quality factor as a function of accelerating gradient for a reduced external magnetic field $(5 \mu \mathrm{T})$. The circle points show $4.5 \mathrm{~K}$ and square ones show $2.4 \mathrm{~K}$. The triangle points are data of intermediate temperature at fixed fields. The dashed line shows $10 \mathrm{~W}$ power consumption and the star is the nominal point.

results evidence that $a Q$ is generated by an external field applied during cavity cooling down near $T_{\mathrm{c}}$.

Note that the external magnetic fields were fully trapped by this cavity. This was checked by flux-gate sensors around the cavity, showing no flux expulsion when the cavity was cooled down across $T_{\mathrm{c}}$. In spite of the incomplete Meissner effect at the superconducting transition, once the cavity was superconducting, and an external field was applied, the Meissner flux exclusion was clearly seen by the sensors, and no $Q_{0}$ degradation was observed.

The observed full field trapping during cool down may be explained by the disordered niobium film, which has presumably more pinning centers than bulk niobium cavities. Also, the copper substrate causes a uniform temperature distribution during cool down, and the low thermal gradient does not provide enough flux repulsion force at the phase front. Here, we do not discuss the origin of low efficiency of flux expulsion in more detail. In the following sections, the trapped flux and the ambient fields are not distinguished.

In Fig. 2, another $Q$ appears when the temperature is increased. This effect also exists in Fig. 3 but is overwhelmed by the effect of trapped flux. The $Q_{0}$ measurements at a fixed field provide the temperature dependence of this $Q$.

\section{ANALYSIS}

Based on the raw data, detailed data analyses were carried out as follows. The measured $Q_{0}$ was converted to the average surface resistance $R_{\mathrm{S}}$

$$
R_{\mathrm{s}}=\frac{G}{Q_{0}}
$$

with the geometrical factor $G$ defined by volume and surface integrals of the rf fields in the cavity and angular frequency $\omega$

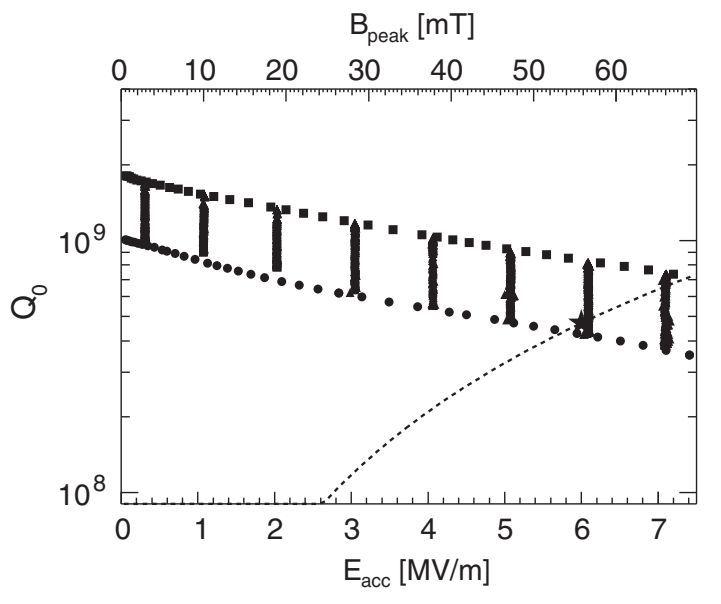

FIG. 3. Quality factor as a function of accelerating gradient for an enhanced external magnetic field $(100 \mu \mathrm{T})$. The circle points show $4.5 \mathrm{~K}$ and square ones show $2.4 \mathrm{~K}$. The triangle points are data of intermediate temperature at fixed fields. The dashed line shows $10 \mathrm{~W}$ power consumption and the star is the nominal point.

$$
G=\frac{\omega \mu_{0} \int_{V} H^{2} d V}{\int_{S} H^{2} d S} .
$$

$G$ was evaluated by commercial codes (CST MicrowAVE STUDIO [11] and HFSS [12]) and is $30.1 \Omega$ for our cavity. When $Q$ exists, Eq. (3) and Eq. (4) should be corrected to take into account the local surface resistance because of the rf field distribution over the cavity surface. However, the correction of this effect does not affect the conclusion of this analysis, and it is omitted in the main text for simplicity. The correction is discussed in Appendix B.

In order to separate the temperature dependent and independent terms, the surface resistance at a given accelerating field was empirically fitted by

$$
R_{\mathrm{s}}(T)=\frac{A}{T} \exp \left(-\frac{\Delta}{k_{\mathrm{B}} T}\right)+R_{\text {res }},
$$

with the three free parameters $A, \Delta$, and $R_{\text {res. }}$. This function is used for interpolating the data for following analysis, and does not necessarily contain any physical meanings. However, it is worth noting that the first term of Eq. (5) is very similar to the linear response of the BCS theory [Eq. (1)] in which the parameters do not depend on the rf field levels.

\section{A. Linear $Q$ in the residual resistance}

Figure 4 compares $R_{\text {res }}$ observed with reduced trapped flux $(5 \mu \mathrm{T})$ to the case when the cavity was cooled down in $100 \mu \mathrm{T}$ ambient field, which was fully trapped, as discussed above. As reported in the literature and also presented recently [13-17], the residual resistance caused by the trapped flux depends on the rf field, and in this study, the dependence is linear to the rf field and substantially influences the $Q$. Consequently, the residual resistance $R_{\text {res }}$ can be fitted by 


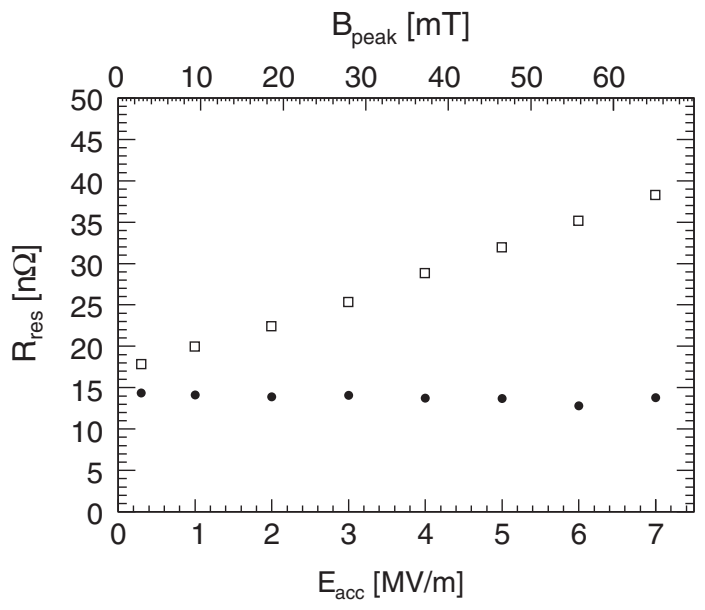

FIG. 4. Residual resistance due to the external field as a function of the accelerating field. The circles show the reduced-field $(5 \mu \mathrm{T})$ cooling result, and blank squares show the data when the ambient field was about $100 \mu \mathrm{T}$.

$$
R_{\text {res }}=R_{\mathrm{s} 0}+R_{\mathrm{s} 1} \times B_{\text {peak }},
$$

where $R_{\mathrm{s} 0}$ and $R_{\mathrm{s} 1}$ are independent of the rf field. The peak magnetic field $\left(B_{\text {peak }}\right)$ on the rf surface was selected as a variable. Whenever the cavity is cooled down without shielding the ambient field, $R_{s 1}$ dominates the total $Q$ at low temperature.

\section{B. Trapped ambient field dependence}

$R_{\mathrm{s} 0}$ and $R_{\mathrm{s} 1}$ are proportional to the trapped field $H_{\mathrm{ext}}$, as shown in Fig. 5. Thus, we can rewrite Eq. (6) as

$$
\begin{aligned}
R_{\text {res }} & =R_{\mathrm{fl}}\left(B_{\text {peak }}, H_{\text {ext }}\right)+R_{\text {res }, 0} \\
& =\left[R_{\mathrm{fl}, 0}+R_{\mathrm{fl}, 1} \times B_{\text {peak }}\right] \times H_{\text {ext }}+R_{\text {res }, 0},
\end{aligned}
$$

where the suffix fl expresses the contribution from trapped flux, and $R_{\text {res }, 0}$ is the intrinsic residual resistance free from

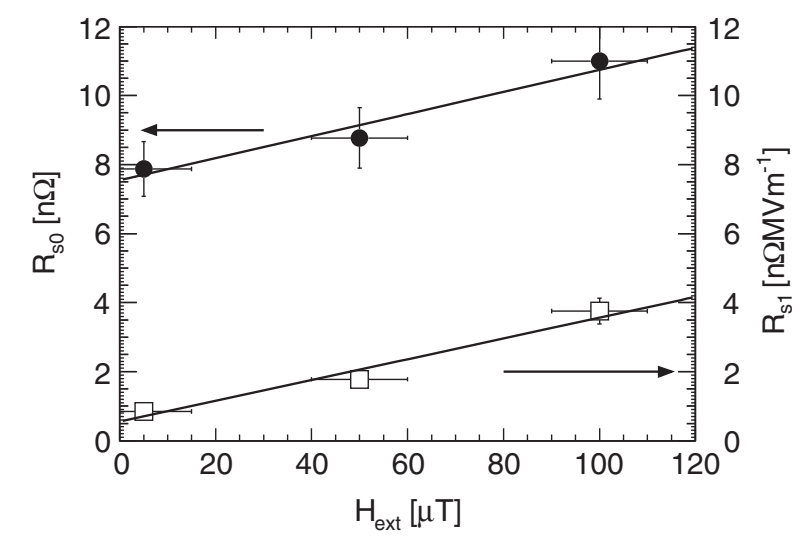

FIG. 5. Residual surface resistances as a function of the trapped magnetic field. The circles show $R_{\mathrm{s} 0}$ and the squares show $R_{\mathrm{s} 1}$.

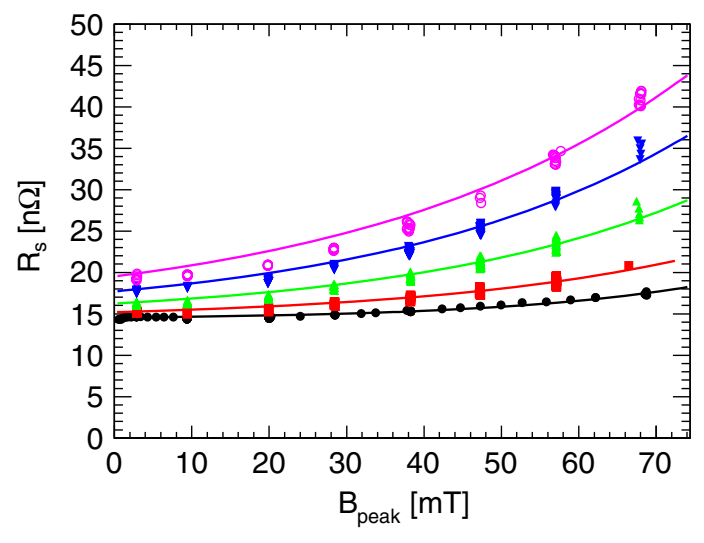

FIG. 6. Temperature dependent surface resistances as a function of the rf field. The corresponding temperatures are 2.4, 3.0, 3.5, 4.0, and $4.5 \mathrm{~K}$ from the bottom. The solid line shows the best empirical fitting at each temperature.

the flux effect. In this study, no dependence of $R_{\text {res }, 0}$ on the rf field was found and $Q$ in $R_{\text {res }}$ was fully due to the trapped flux component $R_{\mathrm{fl}}$. A similar function was reported in another study on $1.5 \mathrm{GHz}$ elliptical $\mathrm{Nb} / \mathrm{Cu}$ cavities [17].

\section{Medium field $Q$}

When the cavity was cooled down under well-compensated ambient fields, the linear $Q$ discussed in Secs. IV A and IV B became negligibly small. In this condition, a different type of $Q$ was exposed, which depends exponentially on the rf field as shown in Fig. 6. Since the temperature dependence of this component is the same as the linear response of an equilibrium BCS state, let us call this $R_{\mathrm{BCS}}^{\prime}\left(T, B_{\text {peak }}\right)$.

The data were heuristically fitted by the function

$$
R_{\mathrm{BCS}}^{\prime}\left(T, B_{\text {peak }}\right)=\frac{A_{0}}{T} \exp \left(-\frac{\Delta_{0}}{k_{B} T}+\alpha B_{\text {peak }}\right),
$$

where $A_{0}$ and $\Delta_{0}$ are fixed at the values determined by the measurement at a low rf field, where linear response Eq. (1) should be still valid, and $\alpha$ is the only free parameter to be determined.

The fitting parameter $\alpha$ turned out to linearly depend on the inverse of the temperature, as shown in Fig. 7. This indicates another empirical parametrization

$$
\alpha=\frac{M}{k_{B} T}
$$

with a new constant $M$ with the dimensions of a magnetic moment. The fitted value of $M$ is

$$
M=1.37(2) \times 10^{-21} \mathrm{JT}^{-1}
$$

Thus, Eq. (9) can be expressed in the form of a reduced gap $\left(\Delta=\Delta_{0}-M B_{\text {peak }}\right)$ version of Eq. (1). 


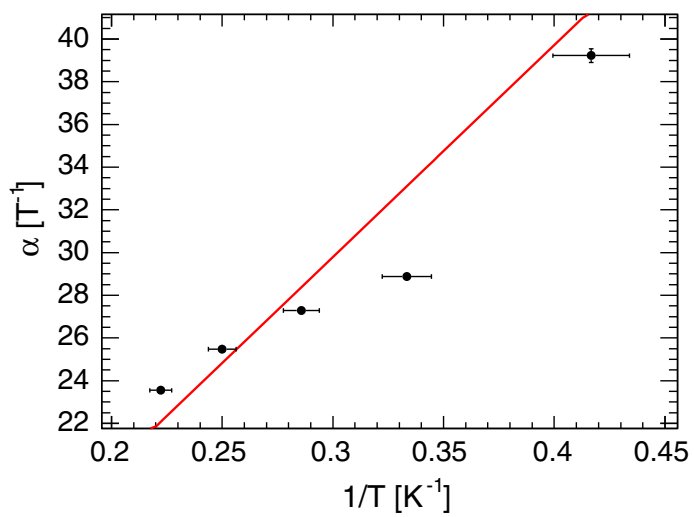

FIG. 7. Temperature dependence of the empirical fitting parameter $\alpha$. The solid line shows the best linear fit without a constant term.

A very similar $Q$ has been observed in bulk niobium cavities and called medium-field $Q$ [18]. A conventional explanation of this $Q$ is a combination of nonlinearities caused by gap reduction and thermal effects [19].

\section{DISCUSSION}

\section{A. $Q$ problem in $\mathrm{Nb} / \mathrm{Cu}$ cavities}

In the above, we have proposed an empirically found formula to fit the surface resistance of $\mathrm{Nb} / \mathrm{Cu}$ cavities

$R_{\mathrm{S}}\left(T, B_{\text {peak }}\right)=R_{\text {BCS }}^{\prime}\left(T, B_{\text {peak }}\right)+R_{\text {fl }}\left(B_{\text {peak }}, H_{\text {ext }}\right)+R_{\text {res }, 0}$,

In our case, each of the three components contributes about one third of the total $R_{\mathrm{S}}$ at the nominal operating condition of the HIE-ISOLDE accelerator as summarized in Table I. Also, two $Q \mathrm{~s}, R_{\mathrm{BCS}}^{\prime}\left(B_{\text {peak }}=60\right)-R_{\mathrm{BCS}}^{\prime}\left(B_{\text {peak }}=0\right)$ and $R_{\mathrm{fl}, 1} \cdot B_{\text {peak }} H_{\text {ext }}$, contribute $(25 \mathrm{n} \Omega)$ more than half of total $R_{\mathrm{s}}$.

This result may shed light on the causes of $Q$ in $\mathrm{Nb} / \mathrm{Cu}$ cavities at low frequency, as it suggests that two environmental conditions $\left(T, H_{\text {ext }}\right)$ are the roots of the problem, which can thus be potentially observed also in bulk niobium

TABLE I. Contribution of each component to $R_{\mathrm{s}}$ at the nominal operating conditions of the HIE-ISOLDE cavities $(T=4.5 \mathrm{~K}$, $\left.H_{\text {ext }}=50 \mu \mathrm{T}, B_{\text {peak }}=60 \mathrm{mT}\right)$.

\begin{tabular}{lcl}
\hline \hline contribution & {$[\mathrm{n} \Omega]$} & \\
\hline$R_{\mathrm{BCS}}^{\prime}$ & 20 & $\begin{cases}R_{\mathrm{BCS}} \equiv R_{\mathrm{BCS}}^{\prime}\left(B_{\text {peak }}=0\right) & 5 \\
R_{\mathrm{BCS}}^{\prime}\left(B_{\text {peak }}=60\right)-R_{\mathrm{BCS}} & 15\end{cases}$ \\
$R_{\mathrm{fl}}$ & 12 & $\begin{cases}R_{\mathrm{fl}, 0} H_{\text {ext }} & 2 \\
R_{\mathrm{fl}, 1} B_{\text {peak }} H_{\text {ext }} & 10\end{cases}$ \\
$R_{\text {res }, 0}$ & 15 & \\
total & 47 & \\
specification & 65 & \\
\hline \hline
\end{tabular}

cavities. Indeed, some studies on bulk niobium cavities near $4.5 \mathrm{~K}$ show similar $R_{\mathrm{BCS}}^{\prime}\left(T, B_{\text {peak }}\right)$ [20] and others at $2 \mathrm{~K}$ with trapped flux also show similar $R_{\mathrm{fl}}\left(B_{\text {peak }}, H_{\text {ext }}\right)$ [21].

Historically, $\mathrm{Nb} / \mathrm{Cu}$ cavities were operated at $4.5 \mathrm{~K}$ without magnetic shields. Superfluid helium cooling was not considered necessary because of the good thermal conductivity of a copper substrate, and because the BCS resistance at low field was optimized by the lower mean free path of niobium films as opposed to the bulk. Magnetic shields were also omitted for coated cavities, because sensitivities to the trapped flux were reported to be small in the literature [17]. However, for our cavity, while the constant term of sensitivity $R_{\mathrm{fl}, 0}$ is indeed one or two orders of magnitude smaller than that of bulk niobium, the linear term $R_{\mathrm{fl}, 1} B_{\text {peak }}$ is not negligible especially when the intrinsic resistance $R_{\text {res }, 0}$ is made smaller by an increased quality of the niobium film.

When the external field was well compensated and the seamless cavity was cooled down by superfluid helium, two $Q$ s were drastically suppressed. The surface resistance smoothly changed when the liquid helium temperature crossing the lambda point. Although detailed field scans were only done up to $B_{\text {peak }}=70 \mathrm{mT}$ as shown in Fig. 2, the cavity reached a maximum $B_{\text {peak }}=120 \mathrm{mT}$ under identical environmental conditions as shown in Fig. 8. This is the highest field value ever achieved in a $\mathrm{Nb} / \mathrm{Cu}$ cavity $[22,23]$. Note that the data were well fitted by Eq. (9) with the same parameter Eq. (10) with the same $M$ in Eq. (11) up to the maximum field without any corrections.

It is worth noting that no low-field $Q$ was observed in this cavity. In a good niobium cavity, surface resistance is usually increased when the rf field is decreased to very low fields. The relatively high residual resistance of our sputtered cavity may prevent the observation of such phenomenon. This is an indirect evidence that the problem of this cavity is not the medium-field $Q$ and flux-induced loss, which

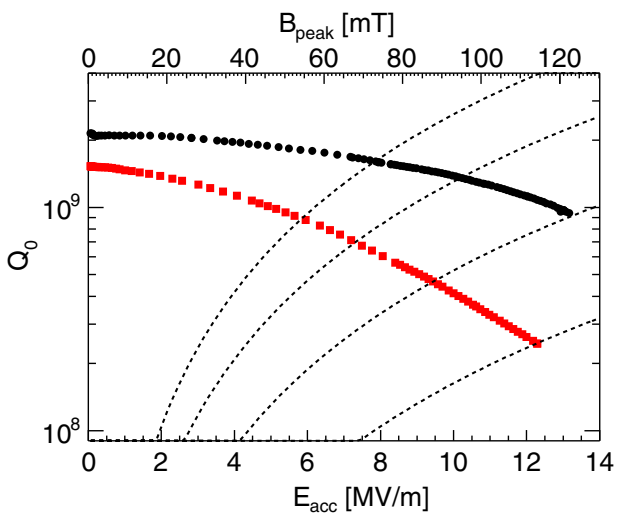

FIG. 8. Maximum peak magnetic field in $\mathrm{Nb} / \mathrm{Cu}$ cavities was achieved when the trapped magnetic field was minimized to $5 \mu \mathrm{T}$. The circle points show $2.4 \mathrm{~K}$ and square ones show $4.5 \mathrm{~K}$. The constant power lines are shown as guidelines, and are $5 \mathrm{~W}$, $10 \mathrm{~W}, 25 \mathrm{~W}$, and $80 \mathrm{~W}$ from the top. 
commonly exist even in bulk niobium cavities, but the intrinsic residual resistance independent of rf fields. Thus, further optimization of the coating parameters should be carried out to reduce the remaining normal conducting defects in the present recipe.

\section{B. Physics interpretation on the trapped flux induced $Q$}

In trying to understand the flux induced components of the surface resistance, we first note that the conventional phenomenological models of the flux oscillation do not predict a linear dependence $R_{\mathrm{fl}, 1}$ on the rf field. The models studied so far are linearized, and their solution, the oscillation amplitude, is proportional to the applied rf field. In such models, the power consumption by the flux oscillation is proportional to the square of the rf field; thus, the surface resistance is constant.

The first model was proposed by Bardeen and Stephen in Ref. [24], where flux motion driven by Lorentz force is described by effective inertial mass, viscosity, and tension. Gittleman and Rosenblum [25] studied trapped fields above $H_{\mathrm{c} 1}$ applied on thin films (PbIn and NbTa alloys of about $12.7 \mu \mathrm{m}$ thick). They neglected the tension term of the Bardeen-Stephen model. Checchin et al. [26] extended this model for pinned flux below $H_{\mathrm{cl}}$ in bulk niobium. They approximated the trapped flux by a flexible tube and the tension term was still omitted. In both cases, the oscillation amplitude of the flux is assumed to be small so that the pinning potential was approximated as harmonic. The approach of Gurevich and Ciovati [27] took into account the tension term, and treated pinning as the Dirichlet boundary condition in which the flux line is fixed at the pinning centers. Still, this model translates into a linear partial differential equation and the predicted surface resistance is independent of the $\mathrm{rf}$ field. Heating at a hot spot caused by the trapped flux was also considered, but the resulting thermal-runaway function does not fit the linearly increasing surface resistance as a function of the rf field.

A natural way to extend these models is to introduce nonlinear terms in the equation of motion. There are several possibilities to do this, for example (1) Collective weak pinning [28] (2) Nonharmonic pinning potential [29] (3) Velocity dependence in the effective viscosity. We compared our data with the collective weak pinning model in Fig. 5(c) of Ref. [28]. The linear dependence of the fluxinduced surface resistance was qualitatively reproduced, but the theoretical curve overestimated the measured data. In the calculation, we used experimentally determined material parameters in Ref. [7]. The depinning current was extracted from the hysteresis curve of the magnetization of a small niobium film sample on a coupon coated in a dummy QWR. The discrepancy might be due to the fact that the measured surface resistance is dominated by the surface property of the film, but the parameters used in the model may be affected by the bulk.
In another model [29], a nonharmonic potential term $1 / 3 \gamma|x|^{3}$ was added to the harmonic potential of Ref. [25]. This qualitatively reproduces the linear dependence on the rf field, but does not provide any physics meanings of $\gamma$; thus, a quantitative comparison of the nonharmonic potential term with our data is not possible. This model also predicts a positive correlation between $R_{\mathrm{fl}, 1}$ and $R_{\mathrm{fl}, 0}$. We do not have enough statistics to address this correlation using the seamless cavities discussed in this paper. However, we found a correlation in the welded cavities. See Appendix C for more discussion.

In summary, there are models which qualitatively predict the linear rf-field dependence we see in our data, but the surface resistance measurement does not provide enough information to support or exclude them, because of the uncertainty in the microscopic model parameters. Direct measurement of the pinned flux may provide some hints on the nonlinearity of the phenomenon [30].

\section{Physics interpretation of medium-field $Q$}

Great care should be taken when attempting physics interpretations of $R_{\mathrm{BCS}}^{\prime}$, although our empirical fitting evokes gap reduction within the BCS framework. Surface resistance is a macroscopic observable averaged over a cavity surface, penetration depth, and rf cycles. Hence, the measured $R_{\mathrm{BCS}}^{\prime}$ is not necessarily a mere microscopic response of the superconductor reacting against the applied rf field. It could arise as well from macroscopic inhomogeneities.

\section{Possible extension of the BCS resistance}

If we ignore the extrinsic effects mentioned above, a possible interpretation of $R_{\mathrm{BCS}}^{\prime}$ may be gap reduction within the extension of the linear response theory.

Depression of superconducting gap by a DC applied magnetic field or current was studied theoretically and experimentally [31-33]. A gap equation modified for a current-carrying state results in an effectively reduced $\Delta$. Tunneling experiments verified the depression of $\Delta$. This phenomenon is more substantial when the sample is thin compared with the penetration depth and when the temperature is close to $T_{\mathrm{c}}$. Besides the gap reduction, the density of states was found to be smeared by a DC current both in dirty and clean limits [34].

A phenomenological model of gap reduction by the $\mathrm{rf}$ current was proposed by Kulik and Palmieri in Refs. [35,36], where $\Delta$ is given by

$$
\Delta=\Delta_{0}-p_{\mathrm{F}} v_{\mathrm{s}}
$$

with $p_{\mathrm{F}}$ the Fermi momentum and $v_{\mathrm{s}} \propto B_{\text {peak }}$ the supercurrent velocity. They substituted this modified $\Delta\left(B_{\text {peak }}\right)$ into Eq. (1). Gurevich recalculated the integral of surface resistance in momentum space followed by averaging over the penetration depth and the rf period [37] rather than using the 
naive substitution. Its result is parabolic in the rf field strength at low field and is exponential at higher rf field. In this model, a characteristic magnetic moment $M$ is

$$
M=\frac{\pi}{2^{3 / 2}} \frac{\Delta}{B_{\mathrm{c}}} \sim 1.13 \times 10^{-22} \mathrm{JT}^{-1} .
$$

This is one order of magnitude smaller than our experimental value Eq. (11). Therefore, we can exclude the model as the cause of the medium-field $Q$ of our cavity. A similar conclusion was reported in a $1.3 \mathrm{GHz}$ elliptical cavity [38]. A possible way to address this discrepancy, which is found in the literature, is to combine thermal feedback with this nonlinear BCS resistance to fit the medium-field $Q$ [18]. This is discussed in the next section.

Apart from the disagreement with the experimental data, the historical approach on the non-linear BCS resistance with the gap reduction deserves some comments motivated by recent theoretical advancements. It was suggested that the gap reduction due to the rf field may be associated with smearing of the density of states $[39,40]$. This phenomenon had been already shown in the DC case in Ref. [35] but was not explicitly used in the derivation of surface resistance. In this context, surprisingly, the gap reduction does exist but is overwhelmed by smearing of the density of states, which reduces the number of effective quasiparticles contributing to the rf loss, and results in a depression of surface resistance, i.e., anti- $Q$. These models were developed to explain the anti$Q$ observed in N-doped cavities [41]. However, their derivation is so general that the gap reduction proposed to explain the normal medium-field $Q$ can be also hidden by the effect of density of states smearing. Note that the formula in Ref. [40] is only valid in the dirty limit, because the Usadel equation [42] is used, but the smearing of the density of states seems a more general phenomenon regardless of the purity of the material as shown in Ref. [34].

These approaches are based on an extension of the gap reduction observed in the DC case to $\mathrm{rf}$ frequencies. Another approach, valid at higher frequency but low field, was proposed by Eliashberg. This leads to the enhancement of $\Delta$ when a high frequency field is applied [43]. The quasiparticle distribution function becomes non-equilibrium by rf photon absorption and effectively $\Delta$ can be larger. Such enhancement was observed experimentally at $10 \mathrm{GHz}$ through tunneling measurements where the current value is very low [44].

The above review illustrates how a field dependence of the effective superconducting gap arises in models going beyond the linear response of the equilibrium BCS state. However, the predictive power of these procedures remains limited. Therefore, we do not conclude that Eq. (9) can theoretically explain the medium-field $Q$ in our case, but just report its exponential rf field dependence, which seems common in low frequency $\mathrm{Nb} / \mathrm{Cu}$ cavities and bulk niobium cavities.

\section{Macroscopic models}

Phenomenologically, there are several possible approaches to explain $R_{\mathrm{BCS}}^{\prime}\left(T, B_{\text {peak }}\right)$ by macroscopic models. Here, we review thermal instability models.

Since the linear response Eq. (1), valid at low rf field, depends on temperature exponentially, Joule heating can cause thermal runaway and can eventually trigger a thermal quench in bulk niobium cavities. However, such thermal runaway does not fit medium-field $Q$-slope, in which the surface resistance gradually increases from low rf fields. To overcome this difficulty, Ref. [18] combines thermal feedback with a nonlinear microscopic theory originating from gap reduction. Figure 9 shows our attempt to use this idea by selecting a reasonable thermal resistance to fit our data. This can fit the $Q$-slope up to $40 \mathrm{mT}$, but not at the higher fields. The curve is qualitatively different from the model. We stress that as discussed in Sec. V C 1, nonlinear extensions of the microscopic theory are still an open field of research, therefore, thermal feedback calculations are associated with high uncertainties.

In addition to the quantitative disagreement, in $\mathrm{Nb} / \mathrm{Cu}$ cavities, thermal instabilities should be strongly suppressed by the good thermal conductivity of copper substrates. In a $1.3 \mathrm{~Hz}$ elliptical cavity, global heating was also excluded by a dedicated experiment measuring the helium gas pressure [38]. Since the frequency of our QWR is about $100 \mathrm{MHz}$, the thermal feedback is even less impacting to the cavity performance. In conclusion, we argue that the conventional thermal feedback models do not explain the medium-field $Q$-slope we observed in this study.

Recently, a new model was proposed, that takes into account defects at the interface between a niobium film and copper substrate [45]. According to this concept, heating may still occur locally on a defect, and a small quenched area has a large influence on the surface resistance, without affecting the average temperature of the cavity surface.

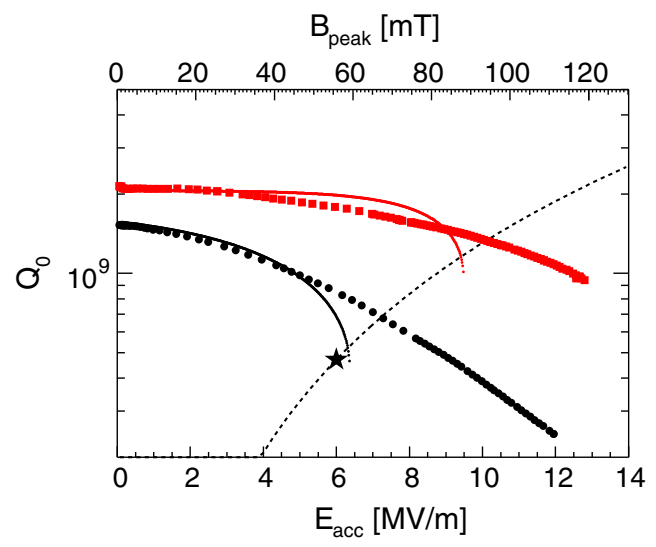

FIG. 9. Comparison of data and thermal feedback of nonlinear $\mathrm{BCS}$ resistance. The black circles show $4.5 \mathrm{~K}$ data and the red squares show $2.4 \mathrm{~K}$ ones. The solid lines are the numerical calculation of the thermal feedback. 


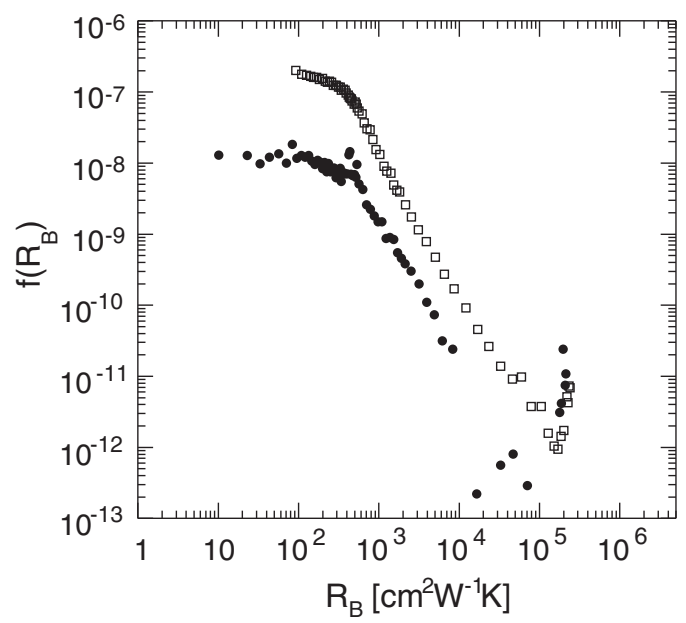

FIG. 10. Converted distribution of thermal boundary resistance. The circle points show $2.4 \mathrm{~K}$ data and blank square ones show $4.5 \mathrm{~K}$ data.

Using a distribution function $f\left(R_{\mathrm{B}}\right)$ of thermal resistance $R_{\mathrm{B}}$ at such defects and local surface resistance $R_{\mathrm{s}}\left(T_{0}, E_{\mathrm{acc}}\right.$, $R_{\mathrm{B}}$ ), which can be quenched by thermal feedback, the observable average surface resistance is given by

$$
\overline{R_{\mathrm{s}}}\left(T_{0}, E_{\mathrm{acc}}\right)=\int_{0}^{\infty} R_{\mathrm{s}}\left(T_{0}, E_{\mathrm{acc}}, R_{\mathrm{B}}\right) f\left(R_{\mathrm{B}}\right) d R_{\mathrm{B}},
$$

where $T_{0}$ is the bath temperature and $R_{\mathrm{B}}$ is a local thermal resistance which locally prevents the film from cooling down. In this model, interface defects with bad thermal resistivity cause local quenches which do not propagate to the rest of the film. At the time of writing this report, this was the only surviving thermal $Q$-slope model for $\mathrm{Nb} / \mathrm{Cu}$ cavities.

A simple algorithm was proposed [46] to inversely solve Eq. (15), and $f\left(R_{\mathrm{B}}\right)$ can be reconstructed from the observed $\overline{R_{\mathrm{S}}}\left(T_{0}, E_{\mathrm{acc}}\right)$. We applied this formalism to the $Q$-slope of the seamless cavity discussed in this paper, and compared two different bath temperatures. Figure 10 shows that calculated $f\left(R_{\mathrm{B}}\right) \mathrm{s}$ differ by one order of magnitude between $2.4 \mathrm{~K}$ and $4.5 \mathrm{~K}$. This is unreasonable because $f\left(R_{\mathrm{B}}\right)$ is dominated by the mechanical adherence of the interface between niobium film and copper substrate, which should not strongly depend on bath temperature.

Previous studies reported that $f\left(R_{\mathrm{B}}\right)$ was independent of the bath temperature $[47,48]$, and concluded that this was an evidence of the thermal boundary problem in $\mathrm{Nb} / \mathrm{Cu}$ cavities. The analysis of one of the authors about the welded QWRs was quoted in these studies. However, these results were obtained without the decomposition of trapped flux effect discussed in Sec. VA. Before the removal of this effect, the linear component $R_{\mathrm{fl}, 1}$ dominates the $Q$-slope. Since $R_{\mathrm{fl}, 1}$ does not strongly depend on temperature, the numerical inversion of Eq. (15) yields $f\left(R_{\mathrm{B}}\right)$ looking independent of temperature on a logarithmic scale.
There are other indirect arguments which may disfavor this model. Thermal simulations showed that defects of small $R_{\mathrm{B}}$, which are abundant in Fig. 10, are not stable above $T_{\mathrm{c}}$ [49]. Also, as emphasized above, a very similar medium-field $Q$-slope has been observed in bulk niobium cavities; thus, a model based particularly on $\mathrm{Nb} / \mathrm{Cu}$ interface may not be reasonable for this $Q$-slope. From these results, we argue that this model in its present form does not also explain the $Q$-slope observed our $\mathrm{Nb} / \mathrm{Cu}$ cavity.

\section{Future prospects of $\mathrm{Nb} / \mathrm{Cu}$ cavities}

This study indicated that the $Q$-slope problem at low frequency $(100 \mathrm{MHz})$ can be avoided if the copper substrate is properly designed, external magnetic fields are well shielded, and bath temperature is low enough, as for bulk niobium cavities. This leads to promising applications of the $\mathrm{Nb} / \mathrm{Cu}$ technology to low- $\beta$ accelerators for heavy ions or upstream sections of proton linear accelerators. For instance, the HIE-ISOLDE post-accelerator is successfully providing radioactive beams for nuclear physics.

The application to higher frequency cavities is still uncertain, because the $1.3 \mathrm{GHz}$ results shown in Ref. [38] could not be decomposed as presented in this paper, and its $R_{\text {res }}$ rather depends exponentially to the applied rf field strength. Historically, the $\mathrm{Nb} / \mathrm{Cu}$ technology has focused on exploring various different coating methods or optimizing deposition parameters. The HIE-ISOLDE experience revealed the overwhelming importance of the copper substrate and supported the choice of a seamless cavity machined from a bulk copper billet.

The good thermal conduction of the seamless QWR also provided uniform temperature distribution during sputtering. This may have some impact on the deposited film quality as well. Clearly, the thermoelectric current and cooldown-dynamics issues deserve more dedicated studies.

\section{CONCLUSION}

The $Q$-slope of a low-frequency $\mathrm{Nb} / \mathrm{Cu} \mathrm{QWR}$ on a seamless substrate was heuristically decomposed into two phenomena. One is responsible for a residual resistance linearly dependent on rf fields, and is caused by the trapped flux. The theory of flux induced loss should be revised because the conventional linearized theories do not contain such rf dependence. If the ambient field is properly shielded, this cavity, whose substrate is machined from bulk copper, is free from this $Q$-slope. The other component is medium-field $Q$-slope which exponentially depends on both rf field and temperature. In superfluid helium, this $Q$-slope is as small as in bulk niobium cavities, and this cavity reached the world best peak magnetic field in the $\mathrm{Nb} / \mathrm{Cu}$ technology. As a similar $Q$-slope was also found in other cavities made of bulk niobium, this component seems a universal property, and not a unique problem of the $\mathrm{Nb} / \mathrm{Cu}$ technology. An empirical fit suggests reduction of $\Delta_{0}$, although a full theoretical 
justification is still missing. Thermal instability models seem not satisfactory. More dedicated studies on medium field $Q$-slope in more controlled environment and cavities are necessary.

\section{ACKNOWLEDGMENTS}

We gratefully acknowledge the contribution of our colleagues A. Sublet, S. Teixeira, T. Mikkola, M. Therasse, and M. A. Fraser for their support in cavity design, preparation, and testing. Our special thanks go to R. Vaglio, M. Arzeo, A. E. Ivanov, and K. Turaj for useful discussion. We warmly thank all the technical staff at CERN for their invaluable help.

\section{APPENDIX A: PERFORMANCE OF THE WELDED CAVITIES}

Figure 11 shows the performance of the welded cavities in the series production [23], obtained in the same experimental conditions as for the seamless cavity discussed in the main text. Unlike for the case of the seamless cavity, we did not see the substantial influence of the trapped ambient field; thus, the results are all for the nominal ambient field of about $50 \mu \mathrm{T}$. Although the cavities selected out of the production cavities met the specification of the cryogenic head load for the HIE-ISOLDE accelerator, the performances of the series production in vertical acceptance tests were sometimes not satisfactory. The difficulty was the quality control of the welding procedure. Note that the $Q$-slope was always there, and was never resolved by magnetic fields or temperature conditions, just as the elliptical cavity result in Ref. [38].

In some of the series production cavities, we found small cracks at the welded section of the copper substrate. These cracks were associated with higher $Q$-slope, and motivated us to design the seamless cavity presented in this paper. As discussed in the main text, surprisingly, the seamless

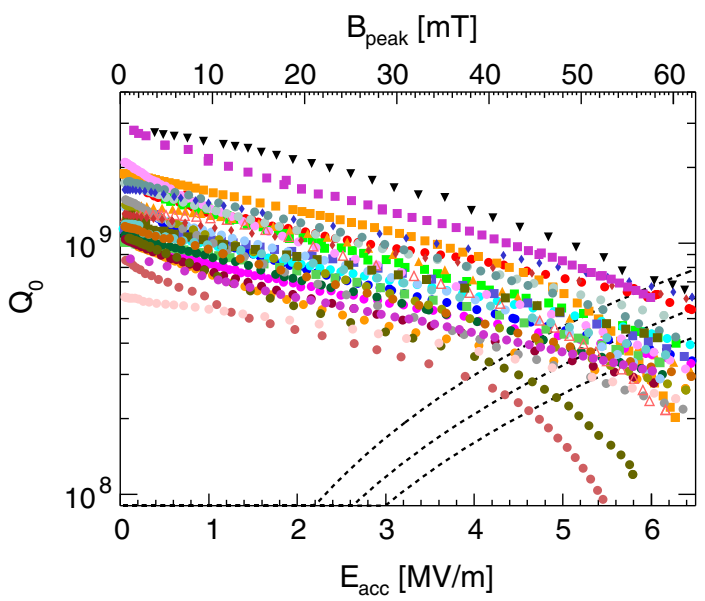

FIG. 11. Quality factor as a function of accelerating gradient in the welded series production cavities. cavity does not just show a better surface resistance but also the performance becomes more controllable by environmental conditions, such as temperature and magnetic fields. It seems that the thermal property of the welded copper substrate hid the physics property of the niobium film. The most substantial change was the cool down effect as discussed in Appendix C.

\section{APPENDIX B: GEOMETRICAL CORRECTIONS}

If the surface resistance varies on the rf surface, the definition of observable surface resistance $\left\langle R_{\mathrm{s}}\right\rangle$ is

$$
\left\langle R_{\mathrm{s}}\right\rangle=\frac{\int_{S} R_{\mathrm{s}}(x, y, z) H^{2}(x, y, z) d S}{\int_{S} H^{2}(x, y, z) d S},
$$

where $R_{\mathrm{S}}$ is the local surface resistance at the given position $(x, y, z)$ and the integral is over the rf surface. If the material is uniform over the surface, the surface resistance does not explicitly depend on position but only through rf field $H$, i.e.,

$$
R_{\mathrm{S}}(x, y, z)=\left(R_{\mathrm{s}} \circ H\right)(x, y, z) .
$$

As D. Longuevergne showed [50,51], one can change the integral variables from $(x, y, z)$ to $H$ with a distribution function $S(H)$ over the surface

$$
\left\langle R_{\mathrm{s}}\right\rangle=\frac{\int_{0}^{H_{\text {peak }}} R_{\mathrm{s}}(H) S(H) H^{2} d H}{\int_{0}^{H_{\text {peak }}} S(H) H^{2} d H} .
$$

Practically, $S(H)$ can be evaluated by electromagnetic simulation codes like CST or HFSS, and the integral of Eq. (B3) can be carried out numerically. In order to obtain $R_{\mathrm{s}}$, one has to invert Eq. (B3) with a given $S(H)$ and measured $\left\langle R_{\mathrm{s}}\right\rangle$. Another study can be found in Ref. [52].

Figure 12 shows the distribution function $S(H)$ of the HIE-ISOLDE QWR studied in this paper. A peak in $S(H)$ means more area in corresponding rf field, i.e., a local plateau of the rf field distribution. There are two peaks in this QWR apart from the peak at the very low field

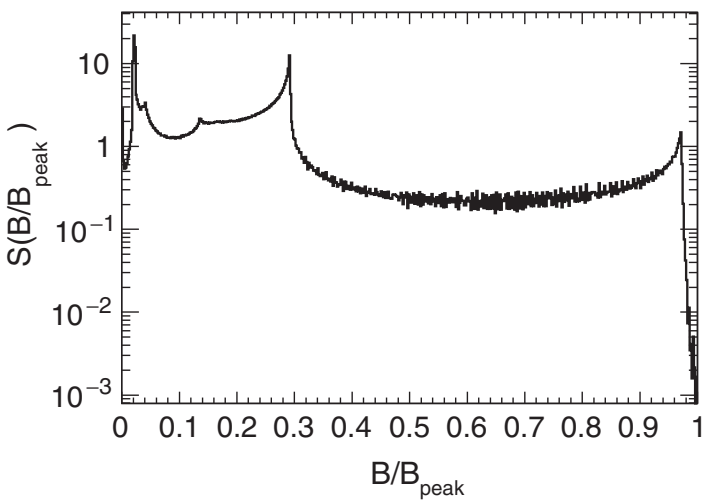

FIG. 12. Area distribution function of $\mathrm{rf}$ field $B$ over the $\mathrm{rf}$ surface as a weight factor of surface integral. 
corresponding to the beam holes. One near the peak field is on the inner conductor, and the other at the medium field exists on the outer conductor. The peaks are smeared by the finite element method, and this lead to zero at the peak field. The rather complicated structure below the peak field contributes to the averaged surface resistance, and the measured $Q$-slope is underestimated from the local $R_{\mathrm{s}}$.

Although aware of this effect, we did not apply the corrections for the analyses in this paper because of the following reasons. The correction does not substantially distort the decomposition by $R_{\mathrm{BCS}}^{\prime}, R_{\mathrm{fl}}$ and $R_{\mathrm{res}, 0}$. The change in fitted $M$ defined by Eq. (10) increases by about only $20 \%$. We did not aim to support or exclude the $Q$ slope models by this precision. Also, this correction is based on the assumption that $R_{\mathrm{s}}$ is uniform over the rf surface. This is not very accurate in our case of a niobium film deposited on a copper substrate. More importantly, trapped flux distribution and its oscillation should be inhomogeneous, which also prevents the simple application of the correction discussed here.

\section{APPENDIX C: COOL DOWN EFFECT IN WELDED CAVITIES}

One complication we faced during the production was cool down dynamics. In the welded cavities [Fig. 1(a)], the inner conductor and outer conductor were machined separately, and welded together after shrink fitting. The nominal penetration depth of the electron-beam welding was $2 \mathrm{~mm}$, with a large spread in the production. Thus, this location became a bottle neck for the heat conduction between inner and outer conductor. As a result, the cavity behavior was completely different from the seamless design shown in Fig. 1(b) even though the coating recipe of the niobium film was the same.

The clear decomposition of $R_{\mathrm{S}}$ proposed in the main text was only possible with the seamless QWR shown in Fig. 1(b) in which the temperature distribution during cooling down was made very uniform by the excellent thermal conductance between the rf surface and the helium bath. The residual resistances of the welded cavities show the similar function shape as described in Eq. (6) obtained for the seamless cavity with trapped flux. Thus, it is natural to suppose that this $Q$-slope is also caused by trapped flux oscillation. However, $R_{\mathrm{s} 1}$ and $R_{\mathrm{s} 0}$ of welded cavities are not influeced by $H_{\mathrm{ext}}$ but affected by the thermal gradient $\Delta T$ between top and bottom of the outer conductor when the cavities are cooled below $T_{\mathrm{c}}$. For these cavities, $R_{\mathrm{s} 1}$ could not be eliminated even by the best possible cooling down in our cryogenic system, and the $R_{\mathrm{BCS}}^{\prime}$ component of those cavities remained masked by the effect of the cool down dynamics.

Both the constant term $R_{\mathrm{s} 0}$ and linear term $R_{\mathrm{s} 1}$ depend linearly on $\Delta T$ and their sensitivities vary for each cavity. Instead of Eq. (8), the flux sensitivity of the welded cavities can be expressed as

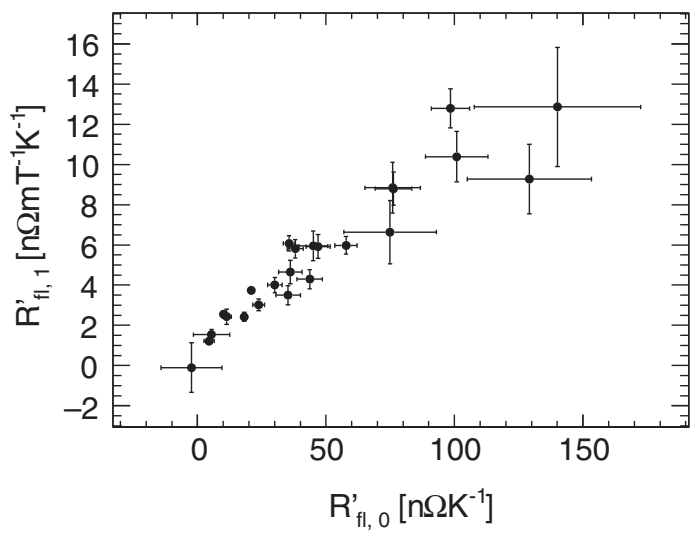

FIG. 13. Correlation plot of $\Delta T$ sensitivity of $R_{\mathrm{s} 1}$ and $R_{\mathrm{s} 0}$.

$$
R_{\mathrm{res}}=\left[R_{\mathrm{fl}, 0}^{\prime}+R_{\mathrm{fl}, 1}^{\prime} \times B_{\text {peak }}\right] \times \Delta T+R_{\mathrm{res}, 0} \cdot,
$$

where $R_{\mathrm{fl}, 0}^{\prime}$ and $R_{\mathrm{fl}, 1}^{\prime}$ are defined as sensitivities to $\Delta T$. Comparing Eq. (8) and Eq. (C1), a natural conclusion is

$$
H_{\text {ext }} \propto \Delta T \text {. }
$$

This indicates an increase of the magnetic trapped flux by thermal gradient. A positive correlation exists between $R_{\mathrm{fl}, 1}^{\prime}$ and $R_{\mathrm{fl}, 0}^{\prime}$ in series production cavities as shown in Fig. 13. This result can be an evidence of the nonharmonic potential model proposed in Ref. [29] and discussed in Sec. V B.

The effect of cool down dynamics in bulk niobium cavities has been intensively studied [53]. Higher thermal gradients are preferred to more efficiently expel the magnetic field upon transition to the superconducting state. This behavior is a clear difference between $\mathrm{Nb} / \mathrm{Cu}$ and bulk niobium, and implies different mechanism on flux-induced surface resistance. One plausible hypothesis is that thermoelectric currents confined in the interface of $\mathrm{Nb} / \mathrm{Cu}$ structure are trapped by the niobium film at $T_{\mathrm{c}}$. Since the Seebeck effect is proportional to the thermal gradient, this hypothesis qualitatively explains the observation Eq. (C1). A similar phenomenon was reported in cavities where the rf surface is a layer of $\mathrm{Nb}_{3} \mathrm{Sn}$ grown on niobium [54]. A direct measurement of such thermoelectric current, however, could not be obtained so far. The required magnetic field to explain the degradation is strong, comparable to or even higher than the earth field. Such current may be locally confined in the interface between them. This may have prevented its direct detection so far. Due to the lack of direct evidence of Eq. (C2), we cannot explicitly support the model [29] in this paper.

[1] C. Benvenuti, N. Cirecelli, and M. Hauer, Niobium films for superconducting accelerating cavities, Appl. Phys. Lett. 45, 583 (1984).

[2] Y. Kadi, Y. Blumenfeld, W. V. Delsolaro, M. A. Fraser, M. Huyse, A. P. Koufidou, J. A. Rodriguez, and F. Wenander, 
Post-accelerated beams at ISOLDE, J. Phys. G 44, 084003 (2017).

[3] J. Bardeen, L. N. Cooper, and J. R. Schrieffer, Theory of superconductivity, Phys. Rev. 108, 1175 (1957).

[4] D. C. Mattis and J. Bardeen, Theory of the anomalous skin effect in normal and superconducting metals, Phys. Rev. 111, 412 (1958).

[5] A. A. Abrikosov, L. P. Gor'kov, and I. M. Khalatnikov, JTEP 8, 182 (1959).

[6] J. Halbritter, Comparison between measured and calculatedRF losses in the superconducting state, Z. Phys. 238, 466 (1970).

[7] A. Miyazaki and W. Venturini Delsolaro, Supercond. Sci. Technol. 32, 025002 (2019).

[8] I. Ben-Zvi and J. M. Brennan, The quarter wave resonator as a superconducting linac element, Nucl. Instrum. Methods Phys. Res. 212, 73 (1983).

[9] S. Teixeira, M. A. Fraser, T. P. Mikkola, A. Miyazaki, and W. V. Delsolaro, CERN Technical Report No. EDMS 1751116, 2017, https://edms.cern.ch/document/1751116/ 2.0 .

[10] S. Teixeira, M. A. Fraser, M. Garlasché, T. Mikkola, A. Miyazaki, A. Sublet, and W. Venturini Delsolaro, A seamless quarter-wave resonator for hie-isolde, in 18th International Conference on RF Superconductivity (JACow, Lanzhou, China, 2017), pp. 686-691.

[11] https://www.cst.com/products/cstmws.

[12] http://www.ansys.com/products/electronics/ansys-hfss.

[13] M. Martinello, Trapped flux sensitivity studies as a function of: Treatment, rf field and frequency, in TTC/ ARIES Topical Workshop on Flux Trapping and Magnetic Shielding (CERN, Switzerland, 2018).

[14] J. Martinello, Overview of flux trapping at Cornell, in TTC/ ARIES Topical Workshop on Flux Trapping and Magnetic Shielding (CERN, Switzerland, 2018).

[15] M. Arzeo, Flux trapping investigation in superconducting samples via the quadrupole resonator, in TTC/ARIES Topical Workshop on Flux Trapping and Magnetic Shielding (CERN, Switzerland, 2018).

[16] K. Umemori, Reports on sensitivity measurements at KEK, in TTC/ARIES Topical Workshop on Flux Trapping and Magnetic Shielding (CERN, Switzerland, 2018).

[17] C. Benvenuti, S. Calatroni, I. Campisi, P. Darriulat, M. Peck, R. Russo, and A.-M. Valente, Study of the surface resistance of superconducting niobium films at $1.5 \mathrm{GHz}$, Physica C (Amsterdam) 316, 153 (1999).

[18] J. Vines, Y. Xie, and H. Padamsee, Systematic trends for the medium field $Q$-slope, in Proceedings of SRF2007 (Peking Univ., Beijing, China, 2007).

[19] H. Padamsee, RF Superconductivity, 1st ed. (WILEYVCH, New York, 2009).

[20] Z. A. Conway, M. P. Kelly, and P. N. Ostoumov, Advanced low-beta cavity development for proton and ion accelerators, Nucl. Instrum. Methods Phys. Res., Sect. B 350, 94 (2015).

[21] G. Ciovati and A. Gurevich, Measurement of RF losses due to trapped flux in a large- grain niobium cavity, in Proceedings of SRF2007 (Peking Univ., Beijing, China, 2007).

[22] W. V. Delsolaro, G. Rosaz, and A. Sublet, CERN Courier 58, 26 (2018).
[23] W. Venturini Delsolaro and A. Miyazaki, Seamless quarter wave resonator for hie-isolde, in 29th Linear Accelerator Conference (JACow, Beijing, China, 2018), pp. 292-296.

[24] J. Bardeen and M. J. Stephen, Theory of the motion of vortices in superconductors, Phys. Rev. 140, A1197 (1965).

[25] J. I. Gittleman and B. Rosenblum, Radio-Frequency Resistance in the Mixed State for Subcritical Currents, Phys. Rev. Lett. 16, 734 (1966).

[26] M. Checchin, M. Martinello, A. Grassellino, A. Romanenko, and J. F. Zasadzinski, Electron mean free path dependence of the vortex surface impedance, Supercond. Sci. Technol. 30, 034003 (2017).

[27] A. Gurevich and G. Ciovati, Effect of vortex hotspots on the radio-frequency surface resistance of superconductors, Phys. Rev. B 87, 054502 (2013).

[28] D. Liarte, D. Hall, P. N. Koufalis, A. Miyazaki, A. Senanian, M. Liepe, and J. P. Sethna, Vortex Dynamics and Losses Due to Pinning: Dissipation from Trapped Magnetic Flux in Resonant Superconducting Radio-Frequency Cavities, Phys. Rev. Applied 10, 054057 (2018).

[29] S. Calatroni and R. Vaglio, Simple model for the rf field amplitude dependence of the trapped flux sensitivity in superconducting rf cavities, Phys. Rev. Accel. Beams 22, 022001 (2019).

[30] L. Embon, Y. Anahory, A. Suhov, D. Halbertal, J. Cuppens, A. Yakovenko, A. Uri, Y. Myasoedov, M. L. Rappaport, M. E. Huber et al., Probing dynamics and pinning of single vortices in superconductors at nanometer scales, Sci. Rep. 5, 7598 (2015).

[31] D. H. Douglass Jr., Magnetic Field Dependence of the Superconducting Energy Gap, Phys. Rev. Lett. 6, 346 (1961).

[32] J. Bardeen, Critical fields and currents in superconductors, Rev. Mod. Phys. 34, 667 (1962).

[33] R. Meservey and D. H. Douglass Jr., Energy gap measurements by tunneling between superconducting films. II. Magnetic field dependence, Phys. Rev. 135, A24 (1964).

[34] P. Fulde, Tunneling density of states for a superconductor carrying a current, Phys. Rev. 137, A783 (1965).

[35] I. Kulik and V. Palmieri, Particle Accelerators 60, 257 (1998).

[36] V. Palmieri, The problem of $Q$-drop in superconducting resonators revised by the analysis of fundamental concepts from RF-superconductivity theory, in Proceedings of SRF2005 (Cornell Univ., Ithaca, NY, US, 2005).

[37] A. Gurevich, Multiscale mechanisms of SRF breakdown, Physica C (Amsterdam) 441, 38 (2006).

[38] T. Junginger, Field dependent surface resistance of niobium on copper cavities, Phys. Rev. ST Accel. Beams 18, 072001 (2015).

[39] B. P. Xiao, C. E. Reece, and M. J. Kelley, Superconducting surface impedance under radiofrequency field, Physica C (Amsterdam) 490, 26 (2013).

[40] A. Gurevich, Reduction of Dissipative Nonlinear Conductivity of Superconductors by Static and Microwave Magnetic Fields, Phys. Rev. Lett. 113, 087001 (2014).

[41] A. Grassellino, A. Romanenko, D. Sergatslov, O. Melnychuk, Y. Trenikhina, A. Crawford, A. Rowe, M. Wong, T. Khabiboulline, and F. Barkov, Nitrogen and argon doping of niobium for superconducting radio 
frequency cavities: a pathway to highly efficient accelerating structures, Supercond. Sci. Technol. 26, 102001 (2013).

[42] N. Kopnin, Theory of Nonequilibrium Superconductivity (Oxford Science Publications, New York, 2001).

[43] G. M. Eliashberg, JETP Lett. 11, 114 (1970).

[44] T. Kommers and C. J., Measurement of MicrowaveEnhanced Energy Gap in Superconducting Aluminum by Tunneling, Phys. Rev. Lett. 38, 1091 (1977).

[45] V. Palmieri and R. Vaglio, Thermal contact resistance at the $\mathrm{Nb} / \mathrm{Cu}$ interface as a limiting factor for sputtered thin film RF superconducting cavities, Supercond. Sci. Technol. 29, 015004 (2016).

[46] S. Calatroni, A. Miyazaki, G. Rosaz, A. Sublet, W. V. Delsolaro, R. Vaglio, and V. Palmieri, Performance analysis of superconducting rf cavities for the CERN rare isotope accelerator, Phys. Rev. Accel. Beams 19, 092002 (2016).

[47] S. Aull, Trapped flux measurements and thermal boundary resistance analysis for an ECR film, in 7th International Workshop on Thin Films and New Ideas for Pushing the Limits of RF Superconductivity (Newport News, VA, US, 2016), https://www.jlab.org/indico/event/156/session/9/ contribution/12.

[48] R. Vaglio and V. Palmieri, Thermal boundary resistance model and defect statistical distribution in $\mathrm{Nb} / \mathrm{Cu}$ cavities, in 18th International Conference on RF Superconductivity (JACow, Lanzhou, China, 2017), pp. 374-377.

[49] H. Furci (private communication).

[50] D. Longuevergne, Magnetic dependence of the energy gap: A good model to fit $Q$ slope of low beta cavities, in 16th International Conference on RF Superconductivity (JACow, Paris, France, 2013), pp. 438-440.

[51] D. Longuevergne, Geometrical corrections for accurate fitting of the field dependent surface resistance for superconducting accelerating cavities, Nucl. Instrum. Methods Phys. Res., Sect. A 910, 41 (2018).

[52] J. Delayen, H. Park, S. De Silva, G. Ciovati, and Z. Li, Determination of the magnetic field dependence of the surface resistance of superconductors from cavity tests, Phys. Rev. Accel. Beams 21, 122001 (2018).

[53] S. Posen, M. Checchin, A. C. Crawford, A. Grassellino, M. Martinello, O. S. Melnychuk, A. Romanenko, D. A. Sergatskov, and Y. Trenikhina, Efficient expulsion of magnetic flux in superconducting radiofrequency cavities for high $Q_{0}$ applications, J. Appl. Phys. 119, 213903 (2016).

[54] S. Posen and D. L. Hall, $\mathrm{Nb}_{3} \mathrm{Sn}$ superconducting radiofrequency cavities: fabrication, results, properties, and prospects, Supercond. Sci. Technol. 30, 033004 (2017). 